\title{
Conocimiento Pedagógico del Contenido: ¿el paradigma perdido en la formación inicial y continua de profesores en Chile?*
}

\author{
Pedagogical Content Knowledge: the missing paradigm in pre-service and in-service \\ science teachers in Chile?
}

\section{Conhecimento Pedagógico do Conteúdo: o paradigma perdido na formação inicial e continuada de professores no Chile?}

\section{Claudia Vergara Díaz, ${ }^{a}$ Hernán Cofré Mardones ${ }^{b}$}

\author{
aUniversidad Alberto Hurtado, Facultad de Filosofía y Humanidades, Santiago, Chile. \\ Correo electrónico: clvergara@uahurtado.cl
}

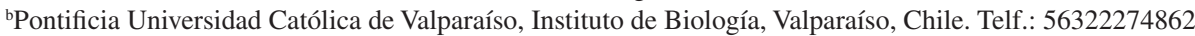

Correo electrónico: hcofrem@hotmail.com

\begin{abstract}
RESUMEN
El conocimiento pedagógico del contenido, CPC (PCK, en inglés) es el constructo teórico más ampliamente utilizado en las últimas décadas en el área de la formación inicial y continua de profesores en todo el mundo. En este artículo, hacemos una revisión crítica y exhaustiva de la literatura del PCK. De manera específica, revisamos los componentes y los diferentes tipos de PCK, la relación entre PCK y la práctica y el efecto del PCK en el aprendizaje de los estudiantes. Finalmente, analizamos el contexto nacional en términos de la formación inicial y continua de profesores en Chile, con especial énfasis en la detección de las oportunidades de desarrollo del PCK.

Palabras claves: conocimiento pedagógico del contenido, CPC, evaluación del PCK, PCK en Chile, formación de profesores.
\end{abstract}

\begin{abstract}
Pedagogical content knowledge (PCK) is the theoretical construct most widely used in the last decades for preservice and in-service teacher training around the world. In this article, we critically and extensively reviewed the international PCK literature. We examine the components and the different PCK styles, the relationship between PCK and practice, and PCK effect on students' learning. Finally, we analyze the national context in relation with pre-service and in-service teachers in Chile, with special emphasis in detecting the opportunities of developing PCK.

Key words: pedagogical content knowledge, PCK, PCK assessment, PCK in Chile, teacher education.
\end{abstract}

\section{RESUMO}

O conhecimento pedagógico do conteúdo CPC (PCK, em inglês) é um dos aportes teóricos mais utilizados nas últimas décadas e em todo o mundo, na área da formação inicial e continuada de professores. Faz-se uma revisão crítica e exaustiva da literatura relacionada ao PCK. Revisam-se os componentes e os diferentes tipos de PCKs, a relação entre PCK e prática e o efeito do PCK sobre a aprendizagem dos estudantes. Por fim, analisa-se o contexto chileno referente à formação inicial e continuada de professores, com ênfase na detecção de oportunidades de desenvolvimento do PCK.

Palavras-chaves: conhecimento pedagógico do conteúdo, CPC, avaliação CPK, CPK no Chile, formação de professores.

Este trabajo fue realizado gracias al proyecto FONDECYT $\mathrm{N}^{\circ} 1131029$ a HC. 
Estudios Pedagógicos, vol. XL, Número Especial 1: 323-338, 2014

CONOCIMIENTO PEDAGÓGICO DEL CONTENIDO: ¿EL PARADIGMA PERDIDO EN LA FORMACIÓN INICIAL Y CONTINUA DE PROFESORES EN CHILE?

\section{INTRODUCCIÓN}

El conocimiento profesional de los profesores puede considerarse como una de las características más importante dentro de la enseñanza (Abell, 2007). Para describir este conocimiento, Shulman (1987) distinguió siete elementos: conocimiento del contenido (SMK), conocimiento del currículo (CUK), conocimiento pedagógico del contenido (PCK), conocimiento pedagógico general (GPK), conocimiento de los estudiantes y sus características (LK), conocimiento del contexto educacional (CK), y el conocimiento de los valores, propósitos y fines de la educación (VAK). Desde que Shulman estableció estas categorías, muchos investigadores han propuesto que el conocimiento pedagógico del contenido (PCK, por su abreviación del inglés pedagogical content knowledge), es el tema más importante en la formación de profesores (ver, por ejemplo, Abell 2007; GessNewsome y Lederman, 1999; Loughran, Berry y Mulhall, 2012), y que niveles mayores de PCK en los profesores podrían propiciar mayores aprendizajes en los estudiantes (Abell, 2007). De hecho, el mismo Shulman (1987) propuso que era dicha categoría la que distinguía al pedagogo del especialista en el contenido, ya que este era el conocimiento específico formado en la intersección del contenido y la pedagogía. Este conocimiento, según Shulman (1986, 1987), implica la comprensión de cómo determinados temas o problemas del contenido a enseñar se organizan, representan y adaptan a los diversos intereses y habilidades de los alumnos.

Este modelo conceptual de la formación de los profesores ha sido ampliamente utilizado en los últimos 20 años (por ejemplo Baumert et al., 2010; Blömeke et al., 2014; Espinosa-Bueno et al., 2001; Gess-Newsome y Lederman, 1993, 1999; Gess-Newsome et al., 1990; Hill, Rowan y Ball, 2005; Loughran, Berry y Mulhall 2012; Loughran, Mulhall y Berry, 2004, 2008; Monte-Sano, 2011; Magnusson, Krajcik y Borko 1999; Olfos, Soto y Silva, 2007; Padilla et al., 2008; Park y Oliver, 2008; Park et al., 2011; Rosenzajn y Yarden, 2014; Schmelzing et al., 2013; van Driel, Verloop y De Vos, 1998) y asociado al concepto de la didáctica específica o didáctica del contenido utilizado en la tradición alemana (van Dijk y Kattmann, 2007), o como conocimiento didáctico del contenido (CDC) o, simplemente, como didáctica en la tradición hispano-francesa (Garritz, 2011). A pesar de esta gran aceptación dentro del mundo académico, la comunidad de investigadores en el ámbito de la formación de profesores aún no ha generado un modelo consistente de PCK, ni tampoco existe consenso a la hora de medir este conocimiento en los profesores (Park y Oliver, 2008). Por otra parte, aún son pocos los estudios que han relacionado directamente este conocimiento con la eficacia de las prácticas de los profesores en formación o en servicio (Olfos, Soto y Silva, 2007; Park et al., 2011) y aún menos los que han determinado empíricamente la manera en que afecta el PCK del profesor sobre el aprendizaje de sus estudiantes (Baumert et al., 2010; Gess-Newsome et al., 2011; Monte-Sano, 2011).

En Chile, la crisis de la educación ha llevado a que las políticas educativas hayan puesto al centro del debate a los profesores, tanto en términos de su evaluación durante su ejercicio profesional (por ejemplo Evaluación docente desde año 2003, AEP desde el año 2002), como a su formación inicial y continua (Cofré, 2010). Dentro de este último tema, han existido valiosos esfuerzos por definir estándares orientadores de la formación docente (MINEDUC, 2012) a cargo de CEPPE y CIAE y evaluar (Programa INICIA, desde el año 2008) aquellos conocimientos relevantes para el logro de una “buena práctica”. Sin embargo, ambos instrumentos se han enfocado, al menos en su declaración explícita, principalmente 
en el conocimiento del contenido (SMK) y el conocimiento pedagógico general (GPK), asumiéndolos como predictores idóneos y suficientes de los conocimientos y capacidades para enseñar del egresado, sin hacer mención explícita del PCK o el manejo de la didáctica específica, a diferencia de países como USA donde este modelo es la base de los Estándares de Desarrollo Profesional de los Profesores (National Research Council, 1994).

Por todo lo anterior, aparece como de suma importancia poder desarrollar una línea de investigación con el objetivo de describir y comprender cuál es el Conocimiento Pedagógico del Contenido (PCK) de los egresados de las distintas carreras de pedagogía en Chile, y cuál es el impacto que éste y otros conocimientos tienen sobre la calidad de las prácticas que realizan y, más aún, en el aprendizaje de sus estudiantes. Desarrollar esta línea de investigación de estudio del PCK fortalecería o coincidiría además con otras dos líneas que necesitan ser desarrolladas con urgencia de acuerdo a las conclusiones del estudio realizado por el CIDE (2007) sobre la investigación educativa en Chile: la formación inicial docente y la didáctica específica. En este artículo se hará una síntesis de los aspectos más importantes dentro de la investigación del PCK en el mundo, en términos de su conceptualización, representación y medición, así como su relación con las prácticas y el aprendizaje de los estudiantes. Más adelante, este marco teórico se contrastará con el contexto nacional, específicamente con los estándares orientadores para la formación de profesores de enseñanza media desarrollados recientemente y los programas de formación de tres carreras de enseñanza media: pedagogía en ciencias, pedagogía en matemáticas y pedagogía en historia. Finalmente, se mostrará un ejemplo de cómo se puede estudiar el desarrollo del PCK para la indagación y la naturaleza de la ciencia en profesores de Biología.

\section{CONCEPTUALIZACIÓN Y REPRESENTACIÓN DEL PCK}

En sus trabajos fundacionales sobre el conocimiento de los profesores, L. S. Shulman $(1986,1987)$ abordó temáticas de crucial importancia en el contexto de la reforma que se vivía en USA hacia mediados de la década de los 80 (para revisiones y análisis del aporte de Shulman puede verse Abell, 2008; Berry, Loughran y van Driel, 2008), sin embargo, el tema que más trascendió fue la creación de un nuevo término en la tradición anglosajona de la enseñanza: el Conocimiento Pedagógico del Contenido o PCK, el cual se ha convertido en uno de los programas de investigación más importantes para el desarrollo del estudio de la formación de profesores y de la enseñanza de las diferentes disciplinas (Abell, 2008; Berry, Loughran y van Driel, 2008; Depaepe, Vershaffel y Kelchtermans, 2013; GessNewsome y Lederman, 1999). Como se dijo anteriormente, pese a su reconocido valor teórico y práctico, el PCK es un concepto esquivo, tanto en su conceptualización como en su medición, aspecto que se discutió desde sus comienzos. En uno de sus primeros planteamientos, Shulman (1986) reconoció la presencia de tres tipos de conocimientos necesarios para la enseñanza: el conocimiento del contenido, el conocimiento del currículo y el PCK. Sin embargo, un año más tarde (Shulman, 1987), este mismo autor reconoció siete categorías básicas de conocimiento (véase introducción). Por otra parte, la definición de PCK de Shulman difiere entre sus escritos (Tabla 1), lo cual ha llevado a diferentes interpretaciones de lo que es el PCK (Figura 1).

Pese a esta confusión y a las subsecuentes controversias sobre su interpretación, medición o coincidencia con otros constructos teóricos como la didáctica específica (véase 
Estudios Pedagógicos, vol. XL, Número Especial 1: 323-338, 2014

CONOCIMIENTO PEDAGÓGICO DEL CONTENIDO: ¿EL PARADIGMA PERDIDO EN LA FORMACIÓN INICIALY CONTINUA DE PROFESORES EN CHILE?

Borowski et al., 2012), el aporte crucial de Shulman fue enfatizar que para enseñar un contenido no basta con saber el contenido y saber de pedagogía general, sino que se deben tener conocimientos específicos de la enseñanza de dicho contenido. Esta proposición ha sido tomada esencialmente en el estudio de la enseñanza de las ciencias (Gess-Newsome y Lederman, 1999; Loughran, Berry y Mulhall, 2012) y las matemáticas (Baumert et al., 2010; Depaepe, Vershaffel y Kelchtermans, 2013; Tirosh, 2000), sin embargo, también existe algún desarrollo de este modelo en el estudio del conocimiento de profesores de historia (McArthur y Bain, 2011; Monte-Sano, 2011; Turner-Bisset, 1999), de inglés como idioma nativo (Howey y Grossman, 1989; Meloth et al., 1989) o de profesores básicos (Nilsson y Loughran, 2012).

Tabla 1. Definiciones de PCK de acuerdo a Shulman (1986) y Shulman (1987)

\begin{abstract}
"Dentro de la categoría del PCK yo incluyo, para los tópicos más regularmente enseñados en un área de contenido, las formas más útiles de representación de estas ideas; las analogías, ilustraciones, ejemplos, explicaciones y demostraciones más poderosos; en pocas palabras, las formas de representación y formulación del tema que lo hace comprensible a otros" (Shulman, 1986: 9).

"El PCK también incluye un entendimiento de lo que hace fácil o difícil el aprendizaje de tópicos específicos: las concepciones y preconcepciones que los estudiantes de diferentes edades y características traen cuando aprenden los tópicos más frecuentemente enseñados. Si estas preconcepciones son errores conceptuales, como lo son frecuentemente, los profesores necesitan el conocimiento de las estrategias que más probablemente funcionen en la reorganización del entendimiento de los estudiantes" (Shulman, 1986: 9).

"Éste (PCK) representa la mezcla entre el contenido y la pedagogía en un esfuerzo por comprender cómo un tópico, problema o tema específico es organizado, representado y adaptado para los diversos intereses y habilidades de los estudiantes y presentado para la enseñanza" (Shulman, 1987: 8).
\end{abstract}

Traducción no literal. La cursiva no es original y destaca los aspectos más importantes de las definiciones.

Trabajos posteriores a Shulman han modificado o especificado ciertos aspectos de la clasificación original de conocimientos profesionales y su relación con el PCK (GessNewsome, 1999; Gess-Newsome et al., 1990; Magnusson, Krajcik y Borko, 1999). Es así como hoy en día se pueden reconocer esencialmente dos modelos de PCK: el integrado y el de transformación (Gess-Newsome, 1999), que aquí hemos llamado complementario (véase también Borowski et al., 2012). Estas dos representaciones se presentan en la Figura 1. En Chile, el modelo complementario de PCK debería ser más útil de adoptar por tres razones fundamentales: la primera es que nos parece que es el que más se acerca a la tradición de la didáctica específica, marco conceptual más arraigado en la educación chilena gracias a la influencia europea, particularmente alemana y española; la segunda es que creemos que es el que mejor rescata la esencia de la propuesta de Shulman de un PCK como un nuevo constructo (véase Tabla 1); y tercero, ya que es el modelo más ampliamente utilizado en los últimos tiempos a través de las diferentes disciplinas de enseñanza (Abell, 2007; Gess-Newsome et al., 1990; Loughran, Mulhall y Berry, 2004; Magnusson, Krajcik y Borko, 1999; Park et al., 2011; Rozenszajn y Yarden, 2014). En este contexto conviene recordar que, por ejemplo, Chevallard (1991) maneja un concepto similar al del PCK: el 
de transposición didáctica (véase por ejemplo Garritz y Trinidad-Velasco, 2004 para una discusión sobre las similitudes de dichos conceptos) y que la tradición alemana posee la "Fachdidaktik" o didácticas de los contenidos disciplinares, la cual se diferencia de la didáctica general y se asemeja al PCK en su característica de disciplina dedicada a estudiar las estrategias específicas de enseñanza de un contenido (van Dijk y Kattmann, 2007). Ambos constructos son más asimilables a un modelo complementario de PCK, el cual se puede diferenciar de los otros dos conocimientos principales de la enseñanza: SMK y GPK.

\section{COMPONENTES, TIPOS Y DESARROLLO DEL PCK}

En la investigación sobre el PCK del profesor se han generado diversas conceptualizaciones, las que han reconocido diferentes componentes dentro de esta categoría de conocimiento (véase Lee y Luft, 2008, para una revisión). Actualmente, se reconocen cuatro subcomponentes principales (Abell 2008; Gess-Newsome et al., 1990; Magnusson, Krajcik y Borko, 1999). Estos conocimientos son: (a) conocimiento sobre las estrategias de enseñanza del contenido específico, (b) conocimiento de la comprensión que tienen los estudiantes de este contenido, (c) conocimiento de las formas de evaluar este contenido, y (d) conocimiento de las metas y objetivos para la enseñanza del contenido en el plan de estudios. De acuerdo a la teoría, los profesores emplean estas categorías de conocimiento de manera integrada para planificar y llevar a cabo la enseñanza (véase también Lee y Luft, 2008). El reconocimiento de estos subcomponentes es un elemento crucial al momento de caracterizar y evaluar el PCK en profesores en formación o ejercicio. Una revisión detallada sobre la investigación de estos cuatro subcomponentes dentro del estudio del PCK de profesores de ciencia, tanto de primaria como secundaria, se puede encontrar en Abell (2007).

Figura 1. Modelos de relaciones entre los dominios del conocimiento profesional del profesor

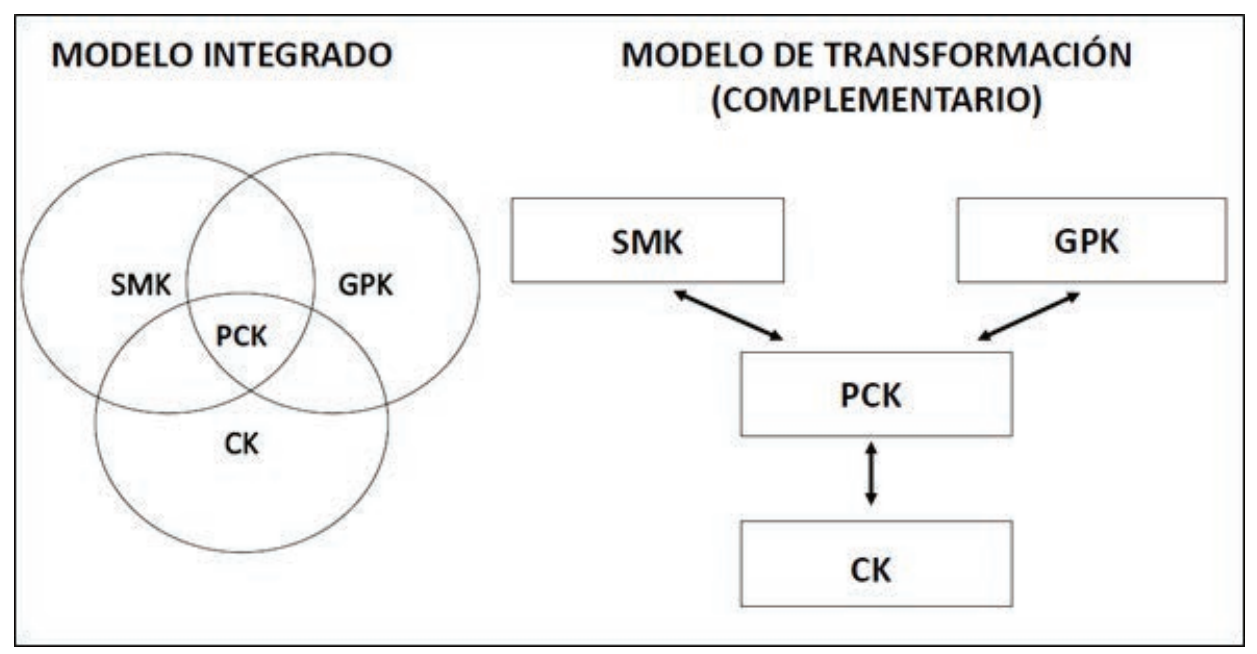


En la Figura 1 se muestra el modelo integrado que entiende el PCK como una “amalgamación” de saberes (Gess-Newsome y Lederman, 1999; Shulman, 1987;), mientras el modelo de transformación enteiende el PCK como un conocimiento independiente, pero complementario a los otros conocimientos necesarios para la enseñanza (Gess-Newsome et al., 1990; Magnusson, Krajcik y Borko, 1999).

Junto a estos componentes, también se han reconocido dos tipos de PCK, el PCK declarativo y el PCK procedimental (Schmelzing et al., 2013). Otros autores han descrito esta separación como el PCK on action (sobre la acción) y el PCK in action (en la acción) (Park y Oliver, 2008), o simplemente saber qué frente a saber cómo (Baumert et al., 2010). El PCK declarativo se puede expresar en textos o entrevistas y corresponde a los conocimientos sobre didáctica específica que posee el profesor, por ejemplo las preconcepciones que mantienen los estudiantes sobre del tema. Por otro lado, el PCK procedimental es una habilidad que tiene que ver con la práctica y con las acciones realizadas durante las clases. Por ejemplo, en esta categoría se inscribe la forma en que el profesor maneja las preguntas de los estudiantes en relación a un contenido específico y la manera en que maneja los errores de los estudiantes. Este conocimiento es básicamente tácito o implícito, por lo que es difícil de transferir, e incluso es difícil de advertir por el mismo profesor (van Driel, Verloop y De Vos, 1998). Debido a la naturaleza distinta de estos dos tipos de PCK, los instrumentos para caracterizarlos o evaluarlos son distintos (ver siguiente sección).

Además de estos subcomponentes y los tipos de PCK, una característica fundamental es su naturaleza dinámica (Abell, 2007, 2008), es decir los profesores desarrollan el PCK con el tiempo a medida que aprenden desde las diferentes oportunidades que enfrentan en su carrera profesional. Diferentes autores (Abell, 2008; Gess-Newsome et al., 1990; Shulman, 1987) han identificado aquellas fuentes a partir de las cuales el PCK se genera y desarrolla. Dentro de estas se pueden reconocer: la observación de las clases, tanto en la etapa de estudiante como en la de profesor-estudiante; la formación disciplinaria; los cursos específicos y materiales propios de la enseñanza de la materia; y la propia experiencia de enseñanza, que parte en las prácticas profesionales, o lo que Shulman, (1987) ha definido como: "the wisdom of practice itself" (la sabiduría de la práctica misma). Si bien algunos autores han señalado a esta práctica de enseñanza como la mayor fuente de desarrollo del PCK (Braund, 2010; Fischler, 2010; van Driel, Verloop y De Vos, 1998), aún existen pocos estudios sobre el desarrollo del PCK, restringiéndose principalmente al desarrollo del PCK en profesores en formación (Barton, McCully y Marks, 2004; Nilsson y Loughran, 2012), y cuyos los resultados han sido contradictorios (pero véase Henze, van Driel y Verloop, 2008; Rozenszajn y Yarden 2014 para estudios sobre el desarrollo de PCK en profesores de ciencia en servicio).

\section{LA MEDICIÓN Y/O CARACTERIZACIÓN DEL PCK}

Como Baxter y Lederman (1999) han señalado, la evaluación del PCK es una tarea compleja que requiere de una combinación de enfoques para mejorar la calidad de la información acerca de lo que los profesores saben, hacen y las razones por las cuales ellos realizan sus acciones. En este sentido, para estudiar y evaluar el desarrollo del PCK es conveniente recopilar datos a partir de múltiples fuentes, entre las que se incluyen 
instrumentos de evaluación de conocimientos en base a cuestionarios de preguntas cerradas o abiertas (Baumert et al., 2010; Blömeke et al., 2014; Gess-Newsome et al., 2011), tareas o reflexiones escritas (Loughran, Mulhall y Berry, 2004; Park y Oliver, 2008; Park et al., 2011; Rozenszajn y Yarden, 2014), observaciones de las clases realizadas por los profesores en formación o en servicio (Berry, 2004; Loughran, Mulhall Berry, 2008; Monte-Sano 2011; Park et al., 2011), entrevistas semi-estructuradas (Monte-Sano, 2011; Park y Oliver, 2008; Park et al., 2011; Rozenszajn y Yarden, 2014), "Stimulated recall" sobre las clases realizadas (Loughran, Mulhall y Berry, 2004), planificaciones de clases (Park et al., 2011; Prescott, Bausch y Bruder, 2013; Rozenszajn y Yarden 2014) y notas de campo (Park y Oliver 2008; Park et al., 2011). Por otra parte, en la caracterización del PCK se ha sugerido distinguir los diferentes subcomponentes que lo forman (Figura 2), y así también evaluarlos mediante las mismas o diferentes metodologías.

Una de las propuestas más utilizadas para este objetivo son los instrumentos CoRes (Content Representation) y PaP-eRs (Pedagogical and Professional experience repertories) desarrollados por John Loughran y sus colegas de la Universidad de Monash (Loughran, Mulhall y Berry, 2004). Estos instrumentos han sido ampliamente utilizados para describir y documentar el PCK de profesores secundarios y primarios, especialmente en profesores de ciencia (Abell, 2008; Espinoza-Bueno et al., 2011; Loughran, Mulhall y Berry, 2004, 2008; Nilsson y Loughran, 2012; Padilla et al., 2008;). Ambos instrumentos fueron desarrollados luego de varios años de trabajar con profesores de ciencia con mucha experiencia. El principal resultado de estos esfuerzos fue el convencimiento de los investigadores de que la mejor forma de caracterizar el PCK era tanto a través de la observación del desempeño del profesor como de entrevistas donde el profesor fuera consultado sobre cómo enseñar y sobre por qué tomó algunas de las decisiones pedagógicas que son observadas.

Figura 2. Componentes del PCK para la enseñanza de la ciencia (modificado de Magnusson, Krajcik y Borko 1999)

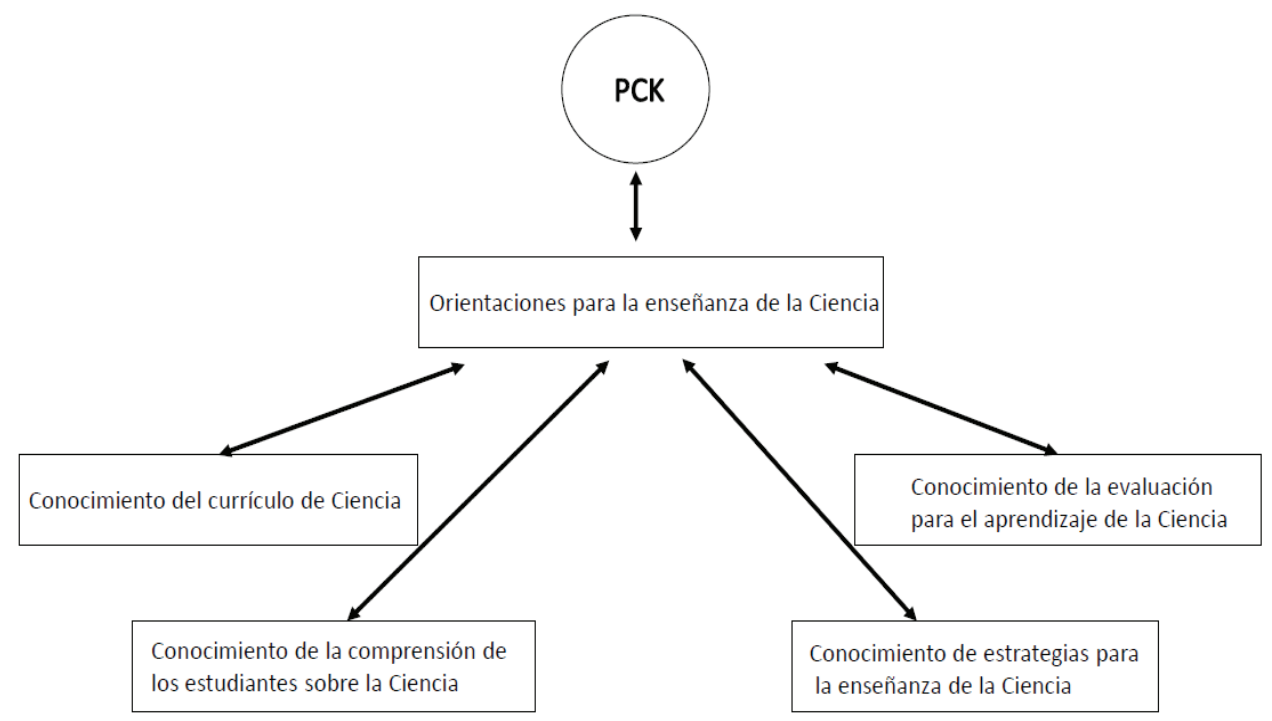


El CoRes es el resultado de una entrevista semi-estructurada en la cual el profesor (o los profesores en grupos pequeños) responde a diversas preguntas que tratan de hacer explícito su PCK sobre algún contenido específico. El instrumento consta de las siguientes preguntas que el investigador debería adaptar para un contenido específico:

1) ¿Cuál es la idea central o más importante que usted espera que aprendan los estudiantes en relación a... (contenido)?

2) ¿Por qué es importante para los estudiantes aprender sobre (contenido)?

3) ¿Qué otras cosas, aparte de la idea central, conoce usted sobre este tema y que no son tan necesarias que los estudiantes aprendan?

4) ¿Cuáles son las dificultades y/o limitaciones que usted reconoce en la enseñanza de (contenido)?

5) ¿Cuáles son las preconcepciones u otras características de los estudiantes que influyen de mayor forma en el aprendizaje de (contenido)?

6) ¿Qué otros factores reconoce que influyen de manera importante en la enseñanza de (contenido)?

7) ¿Qué estrategias conoce que son efectivas para enseñar (contenido) y por qué?

8) ¿Cuáles son las formas específicas con las cuales averigua la comprensión o confusión que los estudiantes tienen respecto a (contenido)?

De esta forma, se llama CoRe al mapa de respuestas generadas por el o los profesores a dichas preguntas. Por ejemplo, a continuación se muestran algunas partes del CoRe generado por un grupo de profesores para la Teoría de Partículas según Loughran, Mulhall y Berry (2004):

Pgta. 1: "que la materia está hecha de pequeñas cosas que llamamos partículas."

Pgta. 4: "que las propiedades macro son el resultado de arreglos micro es algo difícil de entender."

Pgta. 7: "actividades POE (Predecir, Observar y Explicar), por ejemplo, una demostración de agua hirviendo en la cual se explique el porqué del fenómeno."

Por otra parte, el PaP-eRs sirve para ilustrar la forma en que los conocimientos documentados en el CoRe pueden verse reflejados en el acto de enseñar (Loughran, Mulhall y Berry, 2004, 2008). Cada Pap-eRs es el resultado de una reflexión del profesor en relación a un aspecto específico del PCK y de su práctica. Este puede emerger a través de una entrevista de tipo "estimulated recall", en la cual existe una confrontación de los conocimientos documentados en el CoRe, a través del análisis de situaciones de enseñanza videograbadas en relación al contenido tratado por el profesor, o también puede surgir a partir de reflexiones individuales escritas por el profesor en relación a una clase particular (Loughran, Mulhall y Berry, 2004, 2008; Loughran, Berry y Mulhall, 2012). Lo interesante de este grupo de instrumentos es que sirven tanto para caracterizar el PCK declarativo (CoRe), como el procedimental (PaP-eRs). Sin embargo, también existen limitaciones en términos de la cantidad de profesores que se pueden estudiar con este y otras metodologías de tipo cualitativas. En ese sentido, otros autores han preferido desarrollar instrumentos cuantitativos para medir el PCK en temas de biología (Schmelzing et al., 2013) o en temas de matemática (Baumert et al., 2010; Prescott, Bausch y Bruder, 2013).

Algunas proposiciones intermedias han utilizado rúbricas con las que el PCK procedimental es medido evaluando los cuatro subcomponentes que lo forman. La rúbrica 
puede tener diferentes niveles de logro que van desde limitado hasta avanzado. Estas rúbricas pueden ser aplicadas a diferentes tipos de datos: planificaciones, entrevistas y observación de clases. El valor final del PCK se puede generar a través de tres medidas: la suma de los puntajes, el promedio y el mayor valor obtenido. Para describir el desarrollo del PCK de profesores, ambos instrumentos se pueden utilizar en dos momentos temporales distintos (por ejemplo antes o después de un proceso de desarrollo profesional, o antes y después de una experiencia práctica) (Park y Oliver, 2008).

\section{EL PCK, LA PRÁCTICA DOCENTE Y EL DESEMPEÑO DE LOS ESTUDIANTES}

Si bien la cantidad de estudios sobre el PCK de los profesores ha aumentado en los últimos años (Abell, 2007, 2008 para revisiones de PCK en ciencias), todavía es escasa la evidencia empírica que se ha generado en el tema de cómo este conocimiento guía el actuar de los profesores en el aula (Abell, 2007; Baxter y Lederman, 1999). Por otra parte, los pocos estudios que han investigado la traducción del PCK de los docentes a la práctica y a los aprendizajes de sus alumnos han mostrado resultados contradictorios (Baumert et al., 2010; Gess-Newsome et al., 2011; Meloth et al., 1989; Monte-Sano, 2011; Olfos, Soto y Silva, 2007). Uno de los pocos estudios clásicos de PCK y prácticas docentes es el de Meloth et al. (1989), en el cual se describió cómo el PCK sobre lectura de profesores de inglés en formación se evidenciaba en sus clases y, finalmente, influía en la comprensión de lectura de sus estudiantes. Este y otros estudios realizados en profesores de matemática (Olfos et al., 2010) y de biología (Gess-Newsome et al., 2011) han mostrado una falta de relación entre PCK y el aprendizaje de los estudiantes, encontrándose mayores efectos del conocimiento del contenido (SMK) de los profesores sobre el aprendizaje de los estudiantes (Gess-Newsome et al., 2011). No obstante, otros estudios han mostrado que el PCK de profesores de historia, ciencias y matemáticas se relaciona positivamente con sus prácticas (Park et al., 2011) y a su vez con los resultados de aprendizaje de sus estudiantes (Baumert et al., 2010; Monte-Sano, 2011). Estos estudios han mostrado que los profesores con un PCK más sofisticado tienden a crear mejores clases, lo que a su vez tiene un efecto positivo en los aprendizajes de los alumnos (Baumert et al., 2010; Monte-Sano, 2011). Por lo tanto, en términos teóricos, aún existe una necesidad muy grande de investigar la compleja relación entre PCK, prácticas y aprendizaje de los estudiantes (Abell, 2008). De otra parte, uno de los componentes que no se ha estudiado en detalle y que puede estar jugando un rol son las creencias de los profesores sobre la enseñanza y el aprendizaje.

\section{EL DESARROLLO DEL PCK EN EL CONTEXTO NACIONAL}

Existe suficiente evidencia y argumentos para pensar en la didáctica (como disciplina científica) o el PCK (como conocimiento fundamental para la enseñanza de una disciplina) como un ámbito crucial dentro de la formación de profesores. De hecho, uno de los principales aportes del PCK, según varios autores, es su utilidad para comprender y mejorar tanto la formación inicial como continua de los profesores (Abell, 2008; Borowski et al., 2012; Loughran, Berry y Mulhall, 2012). Sin embargo, en Chile aún es muy escasa la 
investigación en torno al PCK en cualquiera de las disciplinas del currículo nacional (pero véase Olfos, Soto y Silva, 2007, para una notable excepción de un estudio en profesores de matemáticas). Por ejemplo, no más del 1,4\% de las investigaciones en educación hasta el año 2007 eran en el área de la didáctica (Corvalán y Ruffinelli, 2007).

Otra forma en que se expresa esta falta de desarrollo del estudio del PCK y de la didáctica como disciplina es su escasa representación en la formación de profesores en Chile, en general, y de manera particular en el área de ciencias. Por ejemplo, si tomamos como referencia las tres disciplinas en que existe más información sobre PCK en la literatura (historia, matemáticas y ciencias) se puede observar que en Chile, dentro de los programas de formación que duran más de cuatro años (es decir excluyendo a los programas de certificación de licenciados y profesionales), el porcentaje del plan de estudios dedicado a cursos de didáctica y prácticas profesionales o finales (oportunidades naturales para desarrollar el PCK), en promedio, nunca sobrepasa el 17\% de la malla en su conjunto (Figura 3).

Figura 3. El gráfico muestra el porcentaje de cuatro diferentes áreas del plan de estudios de tres carreras de pedagogía en Chile

(de este análisis se excluyen los programas de formación para profesionales)

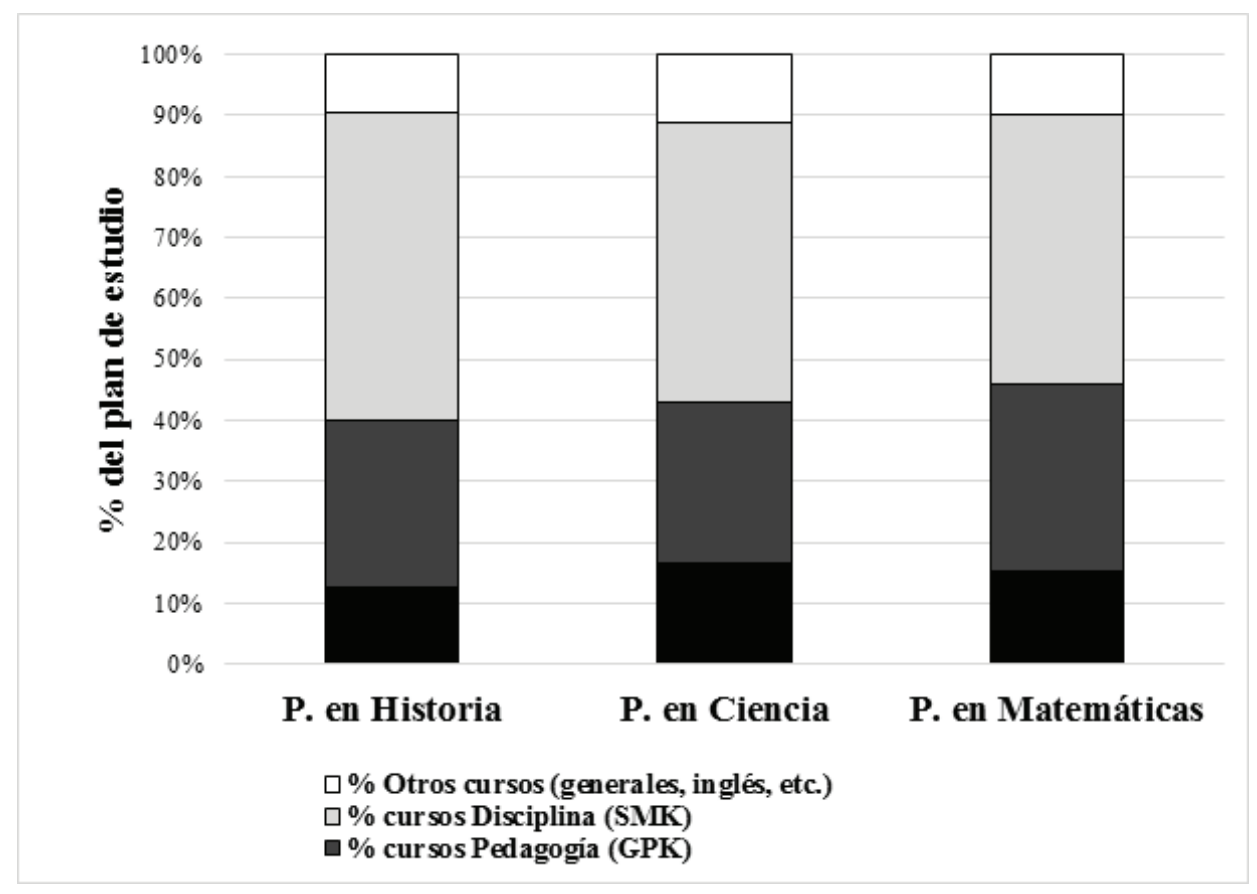

El estudio que se grafica en la Figura 3 fue realizado a comienzos del 2014 e incluyó 32 programas de pedagogía en ciencias (biología, química o física), 20 programas de pedagogía en historia y geografía (o historia y ciencias sociales) y 22 programas de pedagogía en matemáticas. Se reconocieron como cursos donde se desarrolla el PCK aquellos descritos en 
las mallas como didácticas o enseñanza de la especialidad y las prácticas finales, incluyendo aquellas descritas como profesionales y las ubicadas temporalmente justo antes de éstas.

Específicamente, se puede observar que las carreras de pedagogía en ciencias (biología, química y física) son las que presentan el promedio más alto con un $17 \%$ del plan de estudio (con un rango entre un $8 \%$ y un 26\%); luego le sigue la pedagogía en matemáticas, con un promedio de $15 \%$ de la malla de la carrera dedicada al PCK (con un rango entre $8 \%$ y $29 \%$ ), para terminar con la carrera de pedagogía en historia, donde el promedio no sobrepasa el $13 \%$ (con un rango entre $5 \%$ y $25 \%$ ). La magnitud de esta cobertura dentro de los programas de estudio contrasta con lo que sucede con las otras dos áreas asociadas al conocimiento del profesor: el SMK (contenido) y el GPK (pedagogía general) (Figura 3). Como se puede ver en la Figura 3, ambos temas están mucho más representados en la formación de profesores en Chile. El SMK tiene una representación promedio que va desde un 50\% (pedagogía en historia) a un 44\% (pedagogía en matemática) del plan de estudio, mientras que el GPK, tiene una representación que va entre un 31\% (pedagogía en matemáticas) a un $26 \%$ (pedagogía en ciencias) del plan de estudio. Este panorama en la formación de profesores en Chile, contrasta con lo que ocurre en varios países desarrollados con mejores desempeños en las pruebas internacionales como PISA (por ejemplo Alemania, Inglaterra, USA, Italia, Finlandia), en los cuales existe, en la mayoría de las instituciones de formación de profesores, entre un $20 \%$ y un $25 \%$ del plan de estudio dedicado a la enseñanza específica de la disciplina (didáctica) y la formación práctica (Cofré et al., 2010).

Por último, una de las evidencias más claras de la falta de desarrollo en el área de la didáctica y el PCK en los profesores en Chile, tiene que ver con la oferta de postgrados en las diferentes instituciones de educación superior. El análisis de la oferta actual de posgrados en el área de la didáctica de las disciplinas muestra que aún existe mucho que avanzar en la generación de capital humano idóneo para formar a los nuevos profesores de todas las asignaturas (Figura 4). En cuanto a los postítulos y diplomados ofrecidos en el año 2013, de los 97 programas en educación sólo 3 tienen relación con la didáctica ( 1 de ciencias naturales, 1 de matemáticas y 1 de ciencias sociales). Por otra parte, la oferta de postgrados incluye sólo 18 magíster en didáctica de las distintas disciplinas, y son ciencias naturales (6 de ellos) y matemáticas ( 3 de ellos) los más representados, dentro de un universo de 187 programas en educación. De los 13 doctorados del área educativa, sólo 1 (doctorado en didáctica de las matemáticas) es específico en un área de la didáctica. Finalmente, en términos de matrícula al año 2013, sólo el 5,6\% del total de profesores que inició estudios de posgrado, lo hacen en un programa donde podrán desarrollar su conocimiento sobre la enseñanza y el aprendizaje de su disciplina específica (PCK).

Este escenario no deja de ser complejo, no solo por todo lo que hemos revisado anteriormente sobre la importancia del PCK en términos teóricos, sino también por los nuevos desafíos que imponen los nuevos estándares orientadores para la formación de profesores de enseñanza media (CEPPE, 2012). Este documento oficial incluye algunos aspectos relacionados con el PCK, por lo cual es una primera señal de que este componente deberá ser incorporado explícitamente en los planes de estudio de las carreras de pedagogía en el futuro inmediato. En todo caso, un análisis general de los estándares deja en evidencia que tampoco todos los creadores de estos documentos tienen claridad de la importancia de este componente en la formación de profesores, ya que su inclusión es muy desigual dependiendo de la disciplina. 
Figura 4. Oferta de programas de postítulos y posgrados en el área de la educación y el número de ellos dedicado a la didáctica específica en Chile

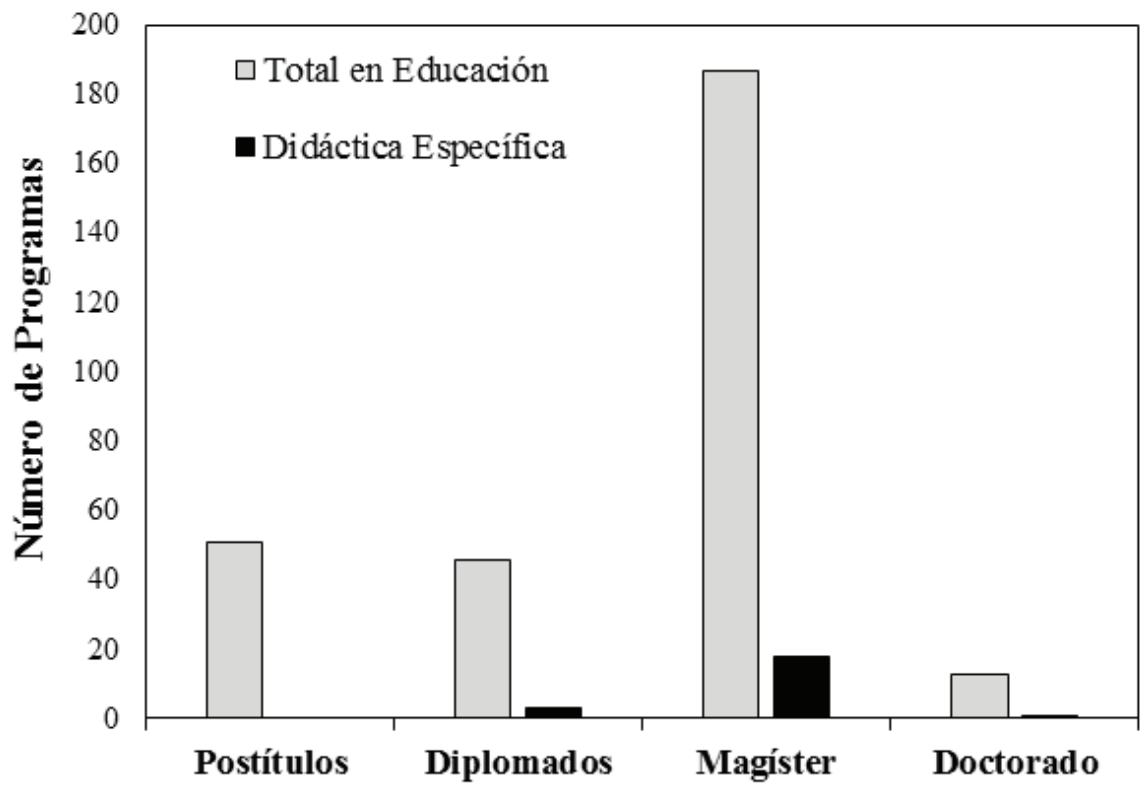

Fuente. Datos obtenidos de índices de posgrados del Consejo Nacional de Educación para el primer semestre 2013

A los 8 estándares de competencias básicas y los 10 estándares de competencias pedagógicos generales (GPK), en la mayoría de las carreras de pedagogía de enseñanza media se deben sumar entre 5 (biología o química) y 11 estándares (historia) asociados al conocimiento de la disciplina (SMK), quedando el PCK reducido a solo 2 o 3 estándares. Mención especial merece el hecho de que la mayoría de los estándares disciplinarios de la formación de profesor de matemática son, en verdad, estándares mixtos, tanto didácticos como disciplinares, en los que no se hace diferencia entre ambos dominios o conocimientos (PCK y SMK).

\section{CONCLUSIONES}

Existe consenso internacional respecto a que el marco teórico del PCK puede ser útil de múltiples formas, impactando principalmente el tema de la formación docente, inicial y continua (Abell, 2008; Loughran, Berry y Mulhall, 2012). Por otra parte, también existe consenso en que aún quedan muchos temas por investigar en este ámbito: la forma en que se desarrolla el PCK, la relación entre PCK del profesor y el aprendizaje de los estudiantes y la relación entre el PCK del académico formador y los profesores principiantes o estudiantes de pedagogía (Abell, 2008). Otros temas relevantes que aún están pendientes son: la relación 
entre el PCK y las creencias sobre enseñanza y aprendizaje de los profesores, la interacción del PCK con otros componentes del conocimiento del profesor, la interacción entre el PCK de profesores principiantes y aquellos de más experiencias, entre muchos otros.

Por otra parte, hemos mostrado evidencias de que el estado actual de nuestra formación de profesores en Chile carece de las instancias suficientes para desarrollar el PCK de los profesores, quedándonos, la mayoría de las veces, en una formación centrada en la disciplina y la pedagogía general. Por otro lado, al mirar la oferta que hacen las universidades, tanto tradicionales como privadas, para la formación continua de profesores en sus diferentes ramas, desde postítulos hasta doctorados, esta tendencia se replica. Es decir, el énfasis está nuevamente en lo pedagógico general y en lo disciplinar.

De lo anterior, se podría pensar que el escenario de la formación de profesores en Chile no es muy alentador y que estamos lejos de poder llegar a lo que nuestra sociedad espera, es decir el mejoramiento de la calidad de la educación en Chile. Sin embargo, otra visión es pensar que tenemos un mundo de oportunidades por delante para mejorar la calidad de la formación de nuestros profesores en formación y en ejercicio. Hemos detectado una gran debilidad y existen las bases teóricas y empíricas que podemos tomar para comenzar a remediar este problema. En este sentido creemos que en el futuro la formación de profesores podría enfocarse en las siguientes líneas de acción:

Primero desde el Estado, a través del Ministerio de Educación y sus diferentes ramas (incluyendo, FONDECYT, CPEIP, entre otros), debería fomentar el perfeccionamiento a nivel de doctorado en las diferentes didácticas específicas con becas exclusivas para este propósito. En la misma línea, se debería incentivar la creación de nuevos programas de magíster o el fortalecimiento de los ya existentes en áreas de didáctica específica. Finalmente, también debería ser una preocupación país el que se generen capacitaciones en las diferentes didácticas específicas para los profesores en ejercicio.

Segundo, las universidades deberían revisar sus planes de estudio para evaluar la posibilidad de incorporar mayores instancias de desarrollo del PCK de los profesores en formación, específicamente incorporando más cursos de didácticas específicas y extendiendo y profundizando las experiencias de prácticas finales. Por otra parte, aquellas universidades que ya tengan una representación importante de estos temas deberían velar por incluir diferentes metodologías para hacer más eficiente y significativas estas instancias, utilizando, por ejemplo, técnicas de microteaching (Gess-Newsome y Lederman, 1993), análisis de video (Fischler, 2010) o el uso de instrumentos como el CoRe y el Pap-eRs para desarrollar le PCK en los profesores en formación (Loughran, Berry y Mulhall, 2012). Además, los investigadores de la didáctica de la disciplina, junto con aquellos responsables de los diferentes programas de formación de profesores, deberían unir esfuerzos y desarrollar investigaciones que apunten hacia la comprensión del fenómeno de desarrollo del PCK de los profesores en formación, así como de aquellos profesores nóveles que se van incorporando todos los años al sistema. Este tipo de iniciativas serviría no solo para comprender mejor el desarrollo del PCK, sino también para apoyar el desempeño de estos profesores en los años más críticos de su profesión.

Esperemos que todos estos desafíos puedan ser implementados en el mediano plazo por los diferentes actores involucrados en la formación de profesores de Chile. Los referentes teóricos están, las presiones por la evaluación y certificación docente están a las puertas, por lo que corresponde que comencemos a realizar de mejor forma la tarea que sin duda nos llevará a mejorar la calidad de todo nuestro sistema educativo. 
Estudios Pedagógicos, vol. XL, Número Especial 1: 323-338, 2014

CONOCIMIENTO PEDAGÓGICO DEL CONTENIDO: ¿EL PARADIGMA PERDIDO EN LA FORMACIÓN INICIAL Y CONTINUA DE PROFESORES EN CHILE?

\section{REFERENCIAS BIBLIOGRÁFICAS}

Abell, S. (2007). Research on science teacher knowledge. In S. Abell \& N. Lederman (Eds.), Handbook of research on science education (pp. 1105-1149). Mahwah, NJ: Lawrence Erlbaum.

Abell, S. (2008). Twenty years later: does pedagogical content knowledge remain a useful idea? International Journal of Science Education, vol.30, n.10, 1405-1416.

Barton, K., McCully, A., \& M. Marks. (2004). Reflecting on elementary children's understanding of History and Social studies: An inquiry project with beginning teachers in Northern Ireland and the United States. Journal of Teacher Education, vol.55, n.1, 70-90.

Baumert, J., Kunter, M., Blum, W., Brunner, M., Voss, T., Jordan, A., Klusmann, U., Krauss, S., Neubrand M., \& Tsai, Y. (2010). Teachers' mathematical knowledge, cognitive activation in the classroom, and student progress. American Educational Research Journal, vol.47, n.1, 133-180.

Baxter, J.A., \& Lederman, N.G. (1999). Assessment and measurement of pedagogical content knowledge. In J. Gess-Newsome \& N.G. Lederman (Eds.), Examining pedagogical content knowledge (pp. 147-161). Dordrecht, The Netherlands: Kluwer Academic Publishers.

Berry, A., Loughran, J., \& Van Driel, J. H. (2008). Revising the roots of pedagogical content knowledge. International Journal of Science Education, vol.30, n.10, 1271-1279.

Blömeke, S., Buchholtz, N., Suhl U., \& Kaiser, G. (2014). Resolving the chicken-or-egg causality dilema: the longitudinal interplay of teacher knowledge and teacher beliefs. Teaching and Teacher Education, vol.37, 130-139.

Borowski, A., Carlson, J., Fischer, H.E., Henze, I., Gess-Newsome, J., Kirschner S., \& Van Driel, J.H. (2012). Different models and methods to measure teachers pedagogical content knowledge. Paper presentado en la reunión anual de la National Association for Research in Science Teaching (NARST), Indianapolis.

Braund, M. (2010). El apoyo al futuro profesor en el contexto de la escuela: en búsqueda de la cooperación en la formación de los profesores de ciencia. En H. Cofré (Ed.), Cómo mejorar la enseñanza de las ciencias en Chile (pp. 113-124). Santiago de Chile: Ediciones Universidad Cardenal Silva Henríquez.

CEPPE. (2012). Estándares orientadores para carreras de pedagogía en educación media. Santiago de Chile: Centro de Estudios de Políticas y Prácticas en Educación, Ministerio de Educación.

Chevallard, Y. (1991). La transposición didáctica. Argentina: AIQUE.

Cofre, H. (Ed.) (2010). Cómo mejorar la enseñanza de las ciencias en Chile. Santiago de Chile: Ediciones Universidad Cardenal Silva Henríquez.

Cofre, H., Camacho, J., Galaz, A., Jiménez, J., Santibáñez, D. y Vergara, C. (2010). La educación científica en Chile: debilidades de la enseñanza y futuros desafíos de la educación de profesores de ciencia. Estudios Pedagógicos, vol.26, n.2, 279-293.

Corvalán, J. y Ruffinelli, A. (2007). Estado del arte de la Investigación y Desarrollo en Educación en Chile. Santiago de Chile: Universidad Alberto Hurtado. Centro de Investigación y Desarrollo de la Educación, CIDE.

Depaepe, F., Vershaffel, L., \& Kelchtermans, G. (2013). Pedagogical content knowledge: a systematic review of the way in which the concept has pervaded mathematics educational research. Teaching and Teacher Education, vol.34, 12-25.

Espinosa-Bueno, J., Labastida, D., Padilla, K. \& Garritz, A. (2011). Pedagogical content knowledge of inquiry: an instrument to assess it and its application to high school in-service science teachers. US-China Education Review, vol.8, n.5, 599-614.

Fischler, H. (2010). Desde el saber hacia la acción: la formación del profesor de ciencia y la práctica de la enseñanza. En H. Cofré (Ed.), Como mejorar la enseñanza de las ciencias en Chile (pp. 85-111). Santiago de Chile: Ediciones Universidad Cardenal Silva Henríquez.

Garritz, A. (2011). Conocimiento didáctico del contenido. Mis últimas investigaciones: CDC en lo afectivo, sobre la estequiometría y la indagación. Revista TED, n.30, 68-81. 
Garritz, A. y Trinidad-Velasco, R. (2004). El conocimiento pedagógico del contenido. Educación Química, vol.15, n.2, 2-6.

Gess-Newsome, J., \& Lederman, N. (1993). Preservice biology teacher's knowledge structures as a function of professional teacher education: a year-long assessment. Science Education, vol.77, n.3, 25-45.

Gess-Newsome, J., \& Lederman, N. (1999). Examining Pedagogical Content Knowledge (Vol. 6). The Netherlands: Springer Press.

Gess-Newsome, J., Cardenas, B.A., Austin, J., Carlson, A.L., Gardner, M., Stuhlsatz, J.A., \& Grossman, P. (1990). In The marking of a teacher: Teacher knowledge and teacher education. New York: Teacher College Press.

Gess-Newsome, J., Cardenas, S., Austin, B., Carlson, J., Gardner, A., Stuhlsatz, M., Taylor J., \& C. Wilson. (2011, April). Impact of educative material and transformative professional development on teachers PCK, practice, and student achievement. Artículo presentado en la Reunión Anual de NARST, Orlando, USA.

Henze, I., Van Driel, J.H., \& Verloop, N. (2008). The development of experienced science teachers' pedagogical content knowledge of models of the solar system and the Universe. International Journal of Science Education, vol.30, n.4, 1321-1342.

Hill, H.C., Rowan, B., \& Ball, D. (2005). Effects of teachers' mathematical knowledge for teaching on student achievement. American Educational Research Journal, vol.42, n.3, 371-406.

Howey, K.R., \& Grossman, P.L. (1989). A study in contrast: sources of pedagogical content knowledge for secondary English. Journal of teacher Education, vol.40, n.5, 24-31.

Lee, E. \& Luft, J.A. (2008). Experienced secondary science teachers' representation of pedagogical content knowledge. International Journal of Science Education, vol.30, n.10, 13431363.

Loughran, J.J., Mulhall, P., \& Berry, A. (2004). In search of pedagogical content knowledge in science: Developing ways of articulating and documenting professional practice. Journal of Research in Science Teaching, vol.41, n.5, 370-391.

Loughran, J.J., Mulhall, P., \& Berry, A. (2008). Exploring pedagogical content knowledge in science teacher education. International Journal of Science Education, vol.30, n.3, 1301-1320.

Loughran, J.J., Berry, A.K., \& Mulhall, P. (2012). Understanding and Developing Science Teachers' Pedagogical Content Knowledge. The Netherlands: Sense Publishers.

McArthur, L., \& Bain, R. B. (2011). Pedagogical content knowledge for world history teachers: What is it? How might prospective teachers develop it? The Social Studies, vol.102, n.1, 9-17.

Magnusson, S., Krajcik, J., \& Borko, H. (1999). Nature, sources, and development of pedagogical content knowledge for science teaching. In J. Gess-Newsome \& N. Lederman (Eds.), Examining Pedagogical Content Knowledge (pp. 95-132). The Netherlands: Science \& Technology Education Library, Kluwer Academic Publishers

Meloth, M.S., Book, C., Putnam J., \& Sivan, E. (1989). Teachers' concepts of reading, reading instruction, and students' concepts of reading. Journal of Teacher Education, vol.40, n.1, 33-39.

Ministerio de Educación [MINEDUC]. (2012). Estándares orientadores para carreras de pedagogía en educación media. Santiago de Chile: CIAE-CEPPE.

Monte-Sano, C. (2011). Learning to open up history for students: preservice teachers emerging pedagogical content knowledge. Journal of Teacher Education, vol.62, n.3, 260-272.

National Research Council. (1994). National science education standards. Washington, DC.: National Academy of Science, National Academy Press.

Nilsson, P., \& Loughran, J. (2012). Exploring the development of pre-service science elementary teachers' pedagogical content knowledge. Journal of Science Teacher Education, vol.23, n.7, 699721.

Olfos, R., Soto, D. y Silva, H. (2007). Renovación de la enseñanza del algebra elemental: un aporte desde la didáctica. Estudios Pedagógicos, vol.33, n.1, 81-100. 
Padilla, K., Ponce de León, A., Rembado, F.M., \& Garritz, A. (2008). Undergraduate professors' pedagogical content knowledge: the case of 'amount of substance'. International Journal of Science Education, vol.30, n.10, 1389-1404.

Park, S., \& Oliver, J. (2008). Revisiting the conceptualization of pedagogical content knowledge (PCK): PCK as a conceptual tool to understand teachers as professionals. Research Science Education, vol.38, n.2, 261-284.

Park, S., Jang, J. \&. Chen, Y., \& Jung, J. (2011). Is pedagogical content knowledge (PCK) necessary for reformed science teaching?: evidence from an empirical study. Research in Science Education, vol.41, n.1, 245-260.

Prescott, A., Bausch, I., \& Bruder, R. (2013). TELPS: A method for analyzing mathematics preservice teachers' pedagogical content knowledge. Teaching and Teacher Education, vol.35, 43-50.

Rosenzajn, R. \& Yarden, A. (2014). Expansion of biology teachers' pedagogical content knowledge (PCK) during a long-term professional development program. Research in Science Education, vol.44, n.1, 189-213.

Shulman, L.S. (1986). Those who understand: knowledge growth in teaching. Educational Researcher, vol.15, n.2, 4-14.

Shulman, L.S. (1987). Knowledge and teaching: foundations of the new reform. Harward Educational Review, vol.57, n.1, 1-22.

Schmelzing, S., Van Driel, J. H., Jüttner, M., Brandenbusch, S., Sandmann, A. \& Neuhaus, B. (2013). Development, evaluation, and validation of a paper-and-pencil test for measuring two components of biology teachers' pedagogical content knowledge concerning the "cardiovascular system". International Journal of Science and Mathematics Education, vol.11, n.6, 1369-1390.

Tirosh, D. (2000). Enhancing prospective teachers' knowledge of children's conceptions: the case of division of fractions. Journal for Research in Mathematics Education, vol.31, n.1, 5-25.

Turner-Bisset, R. (1999). The knowledge bases of the expert teacher. British Educational Research Journal, vol.25, n.1, 39-55.

Van Driel, J. H. Verloop, N., \& De Vos, W. (1998). Developing science teachers' pedagogical content knowledge. Journal of Research in Science Teaching, vol.35, n.6, 673-695.

Van Dijk, E.M., \& Kattmann, U. (2007). A research model for the study of science teachers' PCK and improving teacher education. Teacher and Teaching Education, vol.23, n.6, 885-897. 